\title{
A Hybrid Algorithm for Classification of Compressed ECG
}

\author{
Shubhada S.Ardhapurkar \\ International Center of Excellence in Engineering and Management, Aurangabad, INDIA \\ Email: vaidyashubha21@gmail.com \\ Ramandra R. Manthalkar \\ S.G.G.S. Institute of Engineering and Technology, Nanded, Maharashtra, INDIA \\ Email: rmanthalkar@yahoo.com \\ Suhas S.Gajre \\ S.G.G.S. Institute of Engineering and Technology, Nanded, Maharashtra, INDIA \\ Email: suhasgajre@gmail.com
}

\begin{abstract}
Efficient compression reduces memory requirement in long term recording and reduces power and time requirement in transmission. A new compression algorithm combining Linear Predictive coding (LPC) and Discrete Wavelet transform is proposed in this study. Our coding algorithm offers compression ratio above $85 \%$ for records of MIT-BIH compression database. The performance of algorithm is quantified by computing distortion measures like percentage root mean square difference (PRD), wavelet-based weighted PRD (WWPRD) and Wavelet energy based diagnostic distortion (WEDD). The PRD is found to be below $6 \%$, values of WWPRD and WEDD are less than 0.03 . Classification of decompressed signals, by employing fuzzy c means method, is achieved with accuracy of $97 \%$.
\end{abstract}

Index Terms - Linear Predictive coding, Discrete wavelet transform, Probability Density Function.

\section{Introduction}

The electrocardiogram (ECG) is a graphical representation of heart's functionality and is an important tool used for diagnosis of cardiac abnormalities. A patient monitoring system acquires huge amount of ECG data. ECG data size depends upon several factors like sampling frequency, quantization levels, number of leads and time over which it is recorded. The storage or transmission of ECG demands compression by preserving significant clinical content.

Compression means representing the signal or information with fewer bits by removing redundancy. Compression can be classified into lossless and lossy. In the lossless methods, data is compressed in such a way that the original data is exactly restored upon reconstruction (decompression). In commonly preferred lossy methods, there exists a tolerable data distortion between the original and reconstructed.

Recently several compression methods have been developed with high compression rate and better quality retrieved signal. Yaniv Zigel et al. introduced ECG compression using analysis by synthesis (ASEC) algorithm. The method consists of a beat codebook, long and short-term predictors, and an adaptive residual quantizer [1]. The compression algorithm has efficiently encoded every heart beat, with minimum bit rate, while maintaining a predetermined distortion level. However the procedure yields high computational complexity.

Wavelet-based ECG compression is largely preferred because of its simplicity and high compression performance. Gulay et al. have proposed ECG signal compression by multi-iteration EZW coding for different wavelets and thresholds. Two types of filter banks, wavelet packets (WP) and scosine modulated filter banks, were implemented. Modified embedded zero tree wavelet (MEZW) approach was suggested for better performance [2].

Another algorithm for wavelet based ECG signal coding was found to be efficient in compression, simple in implementation and computation [3]. Manuel BlancoVelasco has verified feasibility of using wavelet packets for compression scheme [4].

A hybrid algorithm consisting Linear Predictive Coding and Wavelet subband coding is proposed in our work. The proposed method provides simple signal processing steps while preserving clinical contents. The paper is organized as follows: Section 2 explains brief theory about LPC and Discrete wavelet transform (DWT). The overall scheme of compression and decompression combined with classification is presented in the same section. Section 3 includes results of our experimentation and discussion.

\section{Material and Methodology}

\subsection{Linear Predictive coding}

Linear prediction coding (LPC) is a method in which a particular value is predicted by linear function of past values of signal. In our proposed ECG compressor, one 
step forward linear predictor which is a FIR filter of order $p$, is used [5]. The predicted value is computed from the equation (1) as

$$
\hat{x}(n)=-\sum_{k=1}^{p} a(k) x(n-k)
$$

where $\hat{x}(n)$ is predicted value, $x(n-1), x(n-2), x(n-3), \ldots$ $x(n-p)$ are past values of signal $x(n)$ and $a(1), a(2), \ldots a(p)$ are filter coefficients. The order $p$ of LPC decides the accuracy of prediction.

The good prediction can be achieved with optimal determination of the filter coefficients. In order to design filter coefficients, coder attempts to minimise mean square error. The error of prediction is expressed as

$e(n)=x(n)-\hat{x}(n)=\sum_{k=0}^{p} a(k) x(n-k)$

where $a(0)=1$. The mean-square value of the error (MSE) is given by equation (3) as

$$
M S E=E\left[e^{2}(n)\right]
$$

where $E[\cdot]$ denotes the mean value operator.

The derivative of MSE with respect to each coefficient is equated to zero. This generates $p$ equations from which coefficients are estimated by autocorrelation or autocovariance method.

$$
\begin{aligned}
& E \sum_{n}\left[e^{2}(n)\right]=\sum_{n}[x(n)-\hat{x}(n)]^{2} \\
& \left.=\sum_{n} x(n)^{2}-2 x(n) \text { 迩 } n\right)+x(n)^{2}
\end{aligned}
$$

$$
\begin{aligned}
& \text { Differentiating and equating to } \\
& \frac{\partial E}{\partial a_{k}}=0 \text { for } 1 \leq k \leq p \\
& \left.\frac{\partial}{\partial a_{k}}\left(\sum_{n} x(n)^{2}-2 x(n) \text { 效 } n\right)+x(n)^{2}\right)=0 \\
& -2 \sum_{n} x(n) \frac{\partial}{\partial a_{k}} \text { 资 }(n)+2 \sum_{n} x(n) \frac{\partial}{\partial a_{k}} x(n)=0 \\
& \left.\sum_{n} x(n) \frac{\partial}{\partial a_{k}} \text { 次 } n\right)=\sum_{n} x(n) \frac{\partial}{\partial a_{k}} x(n)
\end{aligned}
$$

It is clear from equation (1) that

$$
\frac{\partial}{\partial a_{k}} \hat{x}(n)=-x(n-k)
$$

Substituting in equation (5)

$$
\sum_{n} x(n)(-x(n-k))=\sum_{n} \hat{x}(n)(-x(n-k))
$$

$$
\begin{aligned}
& -\sum_{n} x(n) x(n-k)=\sum_{n}\left(-\sum_{i=1}^{p} a_{k} x(n-i)\right)(-x(n-k)) \\
& -\sum_{n} x(n) x(n-k)=\sum_{i=1}^{p} a_{k} \sum_{n} x(n-i) x(n-k) \\
& r_{x x}(k)=\sum_{i=1}^{p} a_{k} r_{x x}(k-i)
\end{aligned}
$$

where $\mathrm{k}=1,2,3 \ldots \mathrm{p}$ and $r_{x x}(k)$ denotes autocorrelation function

Equation (11), using correlation sequences denoted by ' $r_{x x}(k)$ ', represents normal equations or WienerHopf, or Yule-Walker equations. It is structured for the one-step forward predictor. The Levinson-Durbin algorithm gives solution to these normal equations by exploiting special symmetry in the autocorrelation matrix.

\subsection{Discrete Wavelet Transform}

Wavelets transform is extensively preferred for biosignal coding. Wavelet transform observes the signal in time-frequency scope and provides better scale for detecting and removing redundancies.

Discrete wavelet transform (DWT) is associated with scaling functions $\boldsymbol{\varphi ( t )}$ as companion function to the wavelet function. The wavelet function $\psi(\mathbf{t})$ (High pass filter) and scaling function $\boldsymbol{\varphi ( t )}$ (Low pass filter) when convolved with the signal produce detail coefficients (high pass output) and approximation coefficients (low pass output) respectively. Thus first decomposition level is obtained by using two complementary high- and lowpass filters and then half of samples are eliminated by downsampling. The filter's cut off frequency is equal to half of bandwidth of analyzed signal. Therefore a discrete signal $x(n)$ can be expanded at $\mathrm{j}$ level, as follows:

$$
x(n)=c D_{j, k}[x(n)]+c A_{j, k}[x(n)]
$$

Approximations $\mathrm{cA}_{\mathrm{j}, \mathrm{k}}$ correspond to low frequency components present in signal, while details $\mathrm{cD}_{\mathrm{j}, \mathrm{k}}$ contain high frequency components at the $\mathrm{j}$ th level [6]. The $\mathrm{j}$ controls the dilation or contraction of the scale function and $\mathrm{k}$ denotes the position of wavelet function and $\mathrm{n}$ represents the sample number of the $\mathrm{x}(\mathrm{n})$.

Next levels of decomposition of signal $x(n)$ - detail coefficients $\mathrm{cD}_{\mathrm{j}+1}$ and approximation $\mathrm{cA}_{\mathrm{j}+1}$ are obtained in analogous way, where in place of original signal approximation coefficients of $\mathrm{j}$-th decomposition level $\left(\mathrm{cA}_{\mathrm{j}}\right)$ are analyzed. The initial signal is transformed to a set of coefficients containing approximation and $j$ details. Original signal can be reconstructed by up-sampling and passing through the modified LPF and HPFs. For perfect reconstruction same filter coefficients with reverse order are used. Thus wavelet filter constitutes set of four filters called as quadrature mirror filters.

Substituting $\hat{x}(n)$ from equation (1) into (6) 


\subsection{Pre-processing and Compression scheme}

1) Pre-processing: The long ECG record is first divided into excerpts, each of length $\mathrm{N}$ (1024 samples), then denoised by our previously proposed scheme [7]. The pre-processing is basically denoising process which provides better accuracy and improves compression performance. The noise which interferes with the ECG signal may be due to electrode artifacts, line noise, muscle noise and noise due to respiration. The denoising procedure used is presented here:

- $\quad$ ECG is decomposed up to eighth level.

- Baseline wandering is a very low frequency noise, which perturbs the accuracy of analysis of ECG signal. By setting approximation coefficients $\mathrm{cA}_{8}$ (bandwidth below $2 \mathrm{~Hz}$ ) to zero, baseline wander is reduced.

- The high frequency noise is observed mostly at first and second level. Hence detail coefficients $\mathrm{cD}_{1}$ are replaced by zero as these coefficients belong to frequency region above spectral support of ECG.

- Noise embedded in ECG, is further reduced by modeling second level $\mathrm{cD}_{2}$, third level $\mathrm{cD}_{3}$ and forth level $\mathrm{cD}_{4}$ detail coefficients. Cauchy probability density function (PDF) is preferred for better modeling [8]-[10]. The modeling observes these coefficients statistically.

- The small magnitude detail coefficients with large frequency are not defining the morphology of ECG signal and hence can be easily removed. Whereas significant coefficients with less frequency are retained by placing a sliding window over Cumulative distribution function (CDF). The end points of window result in lower and upper thresholds on the coefficients. The coefficients greater than lower threshold but less than upper threshold are set to zero. The signal is reconstructed from new coefficients. The effect of this method is shown in fig. 1
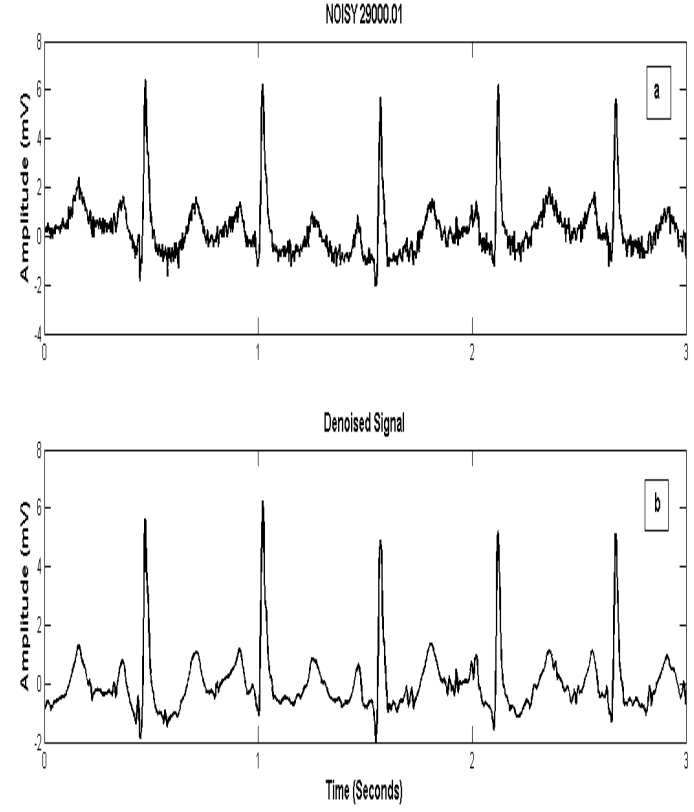

Fig1 (a) Noise contaminated ECG record (b) Denoised ECG

2) Compression Scheme: The total procedure described in the present work has analysis by synthesis structure. In analysis (compressor section) the preprocessed signal is applied as input to the LPC filter of compressor (shown in Fig.2). The error signal is obtained by subtracting output of LPC filter from applied ECG input. The linear prediction filter is essentially all zero filter that generates an output very close to input values. Consequently provides the small amplitude error signal. For this simple reason bits required to represent error signal are less than original with same resolution.

Further compression can be achieved by wavelet filtering. The ECG signal energy is mainly concentrated in the range $3-40 \mathrm{~Hz}$ and so also the range of error (residual) signals. The multiresolution analysis using discrete wavelet transforms helps to separate error signal on frequency basis and removes redundant part.

Error signal ( $\mathrm{N}$ samples) runs through two sections of wavelet filter. The high pass filters output; $c D_{1}$ and $c D_{2}$, of error signal are excessive and do not carry clinical information. Therefore approximation coefficients $c A_{2}$ are considered to define output of compressor. These are fairly sufficient to reconstruct the original signal with better quality. It is evident that, after downsampling at each stage, output $c A_{2}$ contains $\mathrm{N} / 4$ samples.

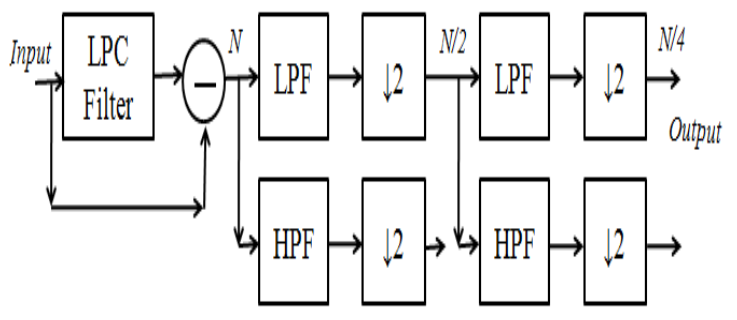

Fig. 2 Compression Scheme (Analysis section) 


\subsection{Classification and decompression}

1) Classification: The compressed signal received at decoder can be classified before decompression by following approach. The two distinct steps involved: feature extraction and Fuzzy-c-means clustering.

Feature extraction: The compact representation of signal pattern is called as feature vector. In the proposed method a feature vector containing four temporal features of compressed segment is used. Temporal features consist of average, minimum, maximum, standard deviation of encoded ECG excerpt. The feature vectors belonging to the records of different classes are applied as inputs to Fuzzy c-means clustering.

Fuzzy-c-means Clustering: Clustering is collection of objects into different groups belonging to certain pattern. Fuzzy clustering allows each feature vector to belong to more than one cluster with different membership degrees (between 0 and 1) and vague or fuzzy boundaries between clusters. This algorithm is based upon iterative optimization of the objective function, with update of membership and cluster centers. Objective function $J_{m}$ is weighted sum of squared errors within groups' and given by

$$
J_{m}=\sum_{i=1}^{N} \sum_{j=1}^{C} u_{i j}^{m}\left\|x_{i}-c_{j}\right\|^{2}, 1<m<\infty
$$

where $m$ is any real number greater than $1, u_{i j}$ is the degree of membership of $x_{i}$ in the cluster $j, x_{i}$ is the $i$ th $d$-dimensional measured data, $c_{j}$ is the $d$-dimension center of the cluster, and $\|\cdot\|$ is any norm expressing the similarity between any measured data and the center.

Firstly $u_{i j}$ are initialised, then centers $c_{i}$ are calculated by equation (14). Then membership $u_{i j}$ are updated by equation (15).

$$
\begin{aligned}
& c_{j}=\left(\sum_{i=1}^{N} u_{i j}{ }^{m} x_{i}\right) /\left(\sum_{i=1}^{N} u_{i j}{ }^{m}\right) \\
& u_{i j}=1 / \sum_{k=1}^{c}\left(\frac{\left\|x_{i}-c_{j}\right\|}{\left\|x_{i}-c_{j}\right\|}\right)^{\frac{2}{(m-1)}}
\end{aligned}
$$

This iteration process stops when $\max \left\{\left|u_{i j}^{(k+1)}-u_{i j}{ }^{k}\right|\right\}<\varepsilon$ where $\varepsilon$ is termination criterion between 0 and 1 whereas $\mathrm{k}$ are iteration steps.

2) Decompression scheme: The decompressor or synthesis section is shown in Fig. 3. It works exactly reverse way that of encoding process. The compressed signal is passed through two sections comprising upsampler and low pass filter. The upsampling process inducts zero after every sample thus fetching high frequency in the signal. The output of inverse wavelet sections is applied to inverse LPC filter (all- pole filter) to reconstruct the original signal.

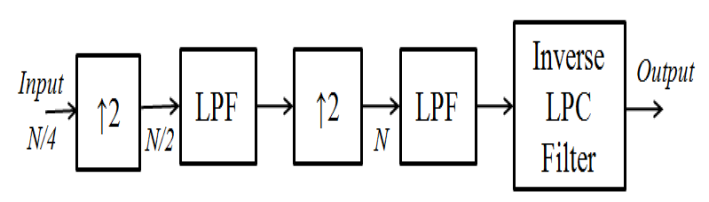

Fig.3 Decompression Scheme (Synthesis section)

\section{Experiments and Analysis}

The proposed method was evaluated on ECG records from MIT-BIH Arrhythmia database and Compression database [11]. The ECG signal excerpt was applied to LPC of encoder. The optimum order of LPC was selected as 4 on the basis of minimum RMS error (RMSE). It is also clear from fig. 4 that a higher order $(p>4)$ increases computational burden and yet does not provide considerable decrease $(0.0126$ to 0.0118$)$ in RMSE.

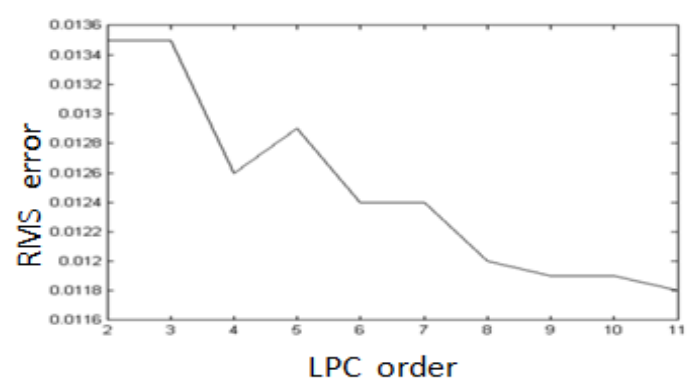

Fig. 4 Root mean square (RMS) error between original and reconstructed signals versus LPC order

The ideal wavelet is one that will encode a signal with greatest possible number of zero or close to zero coefficients. In our experimentation different wavelet filters were applied: Daubechies2, Daubechies4, Daubechies6, bior4.4, bior5.5 and bior6.8. These filters are better choices for ECG processing [12]. Compression performances on several records from MIT-BIH Compression database proved that ' $\mathrm{db} 4$ ' is appropriate choice. Table1. shows results of ECG record 08730_01.

Table1. Compression Performances

\begin{tabular}{|c|c|c|}
\hline Wavelet & RMSE & WWPRD \\
& & \\
\hline $\mathrm{Db} 2$ & 1.6 & 0.11 \\
\hline $\mathrm{Db} 4$ & 1.06 & 0.042 \\
\hline $\mathrm{Db} 6$ & 1.9 & 0.11 \\
\hline Bior 4.4 & 1.45 & 0.125 \\
\hline Bior 5.5 & 1.38 & 0.14 \\
\hline Bior 6.8 & 1.1 & 0.09 \\
\hline
\end{tabular}

$\left({ }^{\mathrm{a}} \mathrm{RMSE}=\mathrm{RMS}\right.$ error, ${ }^{\mathrm{b}} \mathrm{WWPRD}=$ Wavelet based weighted PRD $)$

The root mean square error (RMSE) and WWPRD is given by equation (16) and (19) respectively. 


$$
R M S E=\sqrt{\frac{1}{N} \sum_{n=1}^{N}(x(n)-\tilde{x}(n))^{2}}
$$

where $\mathrm{N}=$ number of samples in the signal, $x(n)=$ original ECG signal, $\tilde{x}(n)=$ reconstructed (decompressed) signal.

The ECG record '12247_01.dat', output of LPC (predicted signal) and the difference (error) signal are shown in Fig. 5. It is obvious to get high error at the beginning due to prediction (Fig. 5 (c)). This variation was completely removed after passing though succeeding wavelet sections (fig.6(c)). The record was selected from MIT -BIH Compression database. This database has 55 records with 20 seconds duration and digitized with sampling frequency 250 samples/sec and 12 bit resolution over10 $\mathrm{mV}$ range. Using these specifications the amplitude and sample number of records were converted into $\mathrm{mV}$ and seconds respectively.
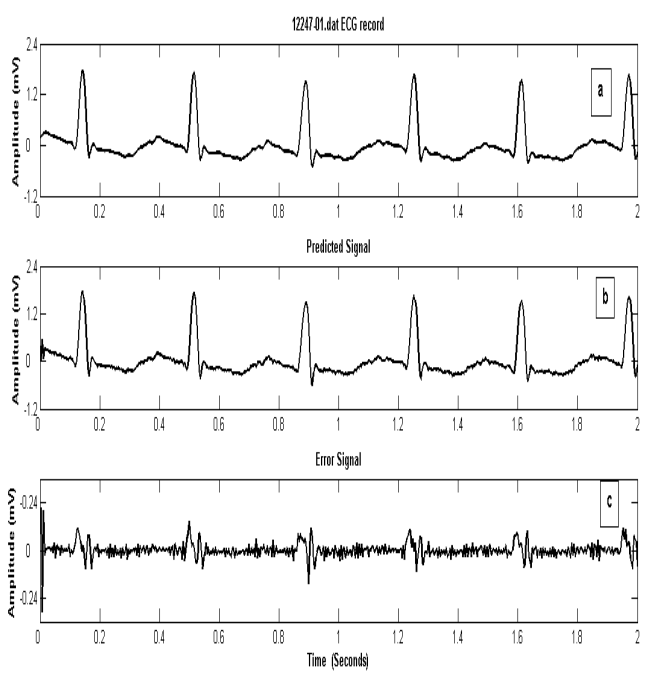

Fig. 5 (a) 12247_01.dat ECG signal of compression database (b) Predicted Signal, (c) Error Signal

The output of compressor is shown in figure 6 .
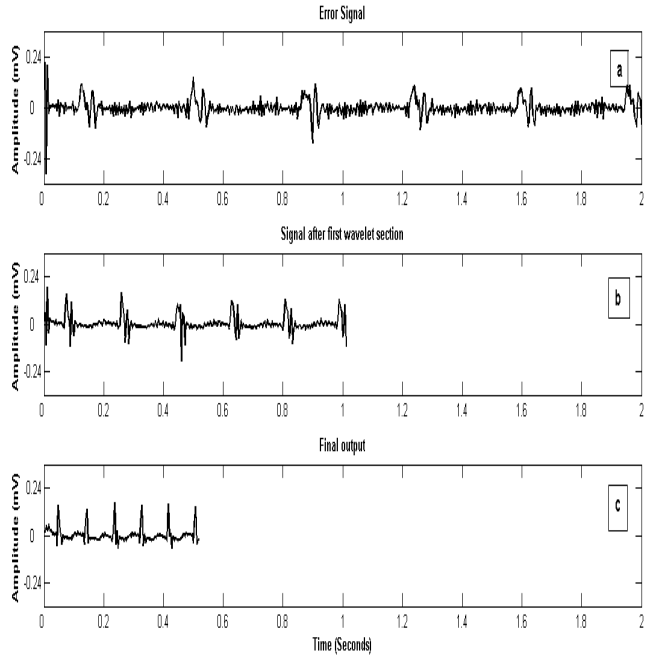

Fig. 6 (a) Error Signal of '12247_01.dat' (b) Approximation Coefficients after first wavelet section (c) Final output,

Sample calculation for compression ratio is explained below:

- 12247 01.dat has absolute maximum magnitude $739(1.80 \mathrm{mV})$ specified with 12 bit resolution (one bit for sign).

- The coder output has absolute maximum magnitude $44(0.10 \mathrm{mV})$. Obviously for binary representation following formula is used.

Number of bits reqd $=\log _{2}(\operatorname{abs}(\max ($ output $)))+1$

$$
=\log _{2}(44)+1=7
$$

Thus 7 bits are required for representation including one bit for sign [3].

- The output signal has one fourth length that of its original signal due to twice downsampling by 2 (refer fig 3).

Therefore compression ratio for ECG signal segment (of $\mathrm{N}$ samples) is given by equation (17).

$$
C R=\frac{N \times 12}{\left(\frac{N}{4}\right) \times N u m b e r \_o f \_b i t s \_r e q d}=\frac{48}{7}=6.85
$$

The above calculation was verified on all records of MIT-BIH Arrythmia database. The proposed algorithm achieved compression ratio $7.33: 1$ for 24 records, $6.28: 1$ for 15 records, 8.8:1 for 7 records and 5.5:1 for 2 records with PRD less than $6 \%$. The quality measure, PRD indicates reconstruction fidelity by point wise comparison with the original data and is given by equation (18). The PRD values were found to be below $3.5 \%$ for 55 records of compression database. The histogram of PRD values is indicated in fig 7.

$$
P R D=100 \times \sqrt{\sum_{n=1}^{N}[x(n)-\tilde{x}(n)]^{2} / \sum_{n=1}^{N}[x(n)]^{2}}
$$

where $\mathrm{N}=$ number of samples in the signal, $x(n)=$ original ECG signal, $\tilde{x}(n)=$ reconstructed (decompressed) signal.

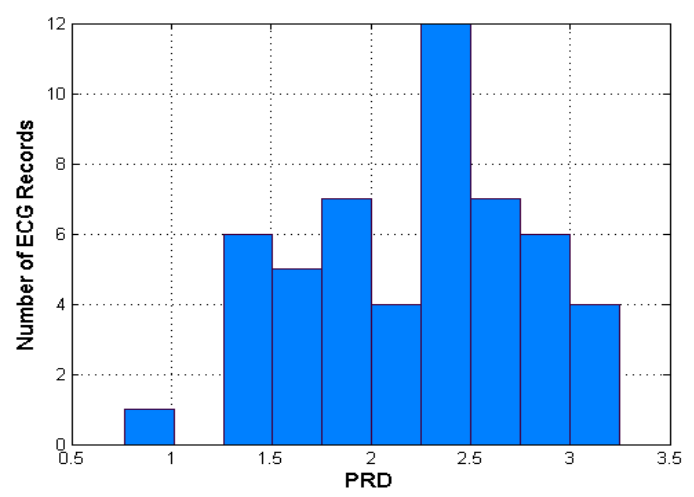

Fig.7 Histogram of PRD values of MIT-BIH compression records. 


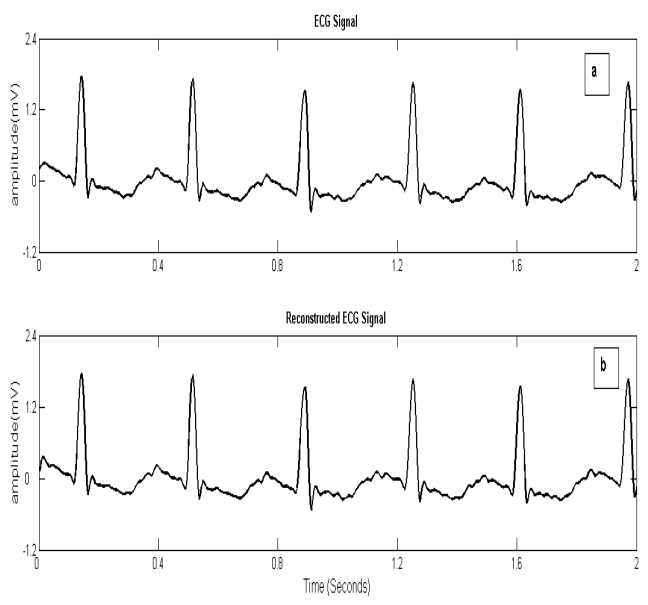

Fig. 8 (a) Input signal '12247_01.dat' (b) Decompressed output

The decompressed signal was found to be very similar to ECG input signal as shown in fig 8. The diagnostic distortion present in the output signal is quantified by finding the distortion measures, Wavelet based weighted PRD (WWPRD) and Wavelet based energy diagnostic distortion (WEDD) [13].

For measuring WWPRD and WEDD, original signal and reconstructed signal were wavelet decomposed to fifth level using ' $\mathrm{Db} 4$ '. The distortion is correlated to subbands. The weighted score associated to each band depends on dynamic range and its diagnostic significance. The PRD value is used as error measure for each band, which is called as WPRD. WWPRD is expressed by equation (19).

$W W P R D=\sum_{j=1}^{L} w_{j} W P R D_{j}$

where $L$ is the number of decomposition level, $\mathrm{w}_{\mathrm{j}}$ is weight associated to $\mathrm{j}^{\text {th }}$ subband given by equation $(20)$. $W{ }^{2} D_{j}$ is the PRD value of the $j^{\text {th }}$ subband expressed by equation (21).

The weight of each subband is calculated as the ratio of sum of the absolute value of coefficients within that band and the sum of absolute value of wavelet coefficients in all the bands .

$$
w_{j}=\left(\sum_{k=1}^{K_{l}}\left|d_{l}(k)\right|\right) /\left(\sum_{m=1}^{L+1} \sum_{k=1}^{K_{l}}\left|d_{m}(k)\right|\right)
$$

$$
W P R D_{j}=\sqrt{\sum_{k=1}^{K_{l}}\left[d_{l}(n)-\tilde{d}_{l}(n)\right]^{2} / \sum_{k=1}^{K_{l}}\left[d_{l}(n)\right]^{2}}
$$

where $d_{l}(k)$ represents detail coefficient at $l^{\text {th }}$ level and $K_{l}$ denotes total number of coefficients at $l^{\text {th }}$ level.

The wavelet transform describes signals in terms of coefficients, representing their energy content in a specified time-frequency region. Basically, the QRS, P and $\mathrm{T}$ waves occupy different subbands with different energy. QRS complex in the ECG signal mostly has the largest amplitude and prominent in the second and third scales. The energies of $\mathrm{T}$ and $\mathrm{P}$ waves are mainly at scales of four and five. Wavelet based energy diagnostic distortion WEDD measure proves that errors in the diagnostic features are reflected in concerned subbands. WEDD is described by equation (22) and associated weights are defined by equation (23).

$$
\begin{gathered}
\text { WEDD }=\sum_{j=1}^{L+1} w_{j} W R_{j} D_{j} \\
w_{j}=\left(\sum_{k=1}^{K_{l}} d_{l}^{2}(k)\right) /\left(\sum_{m=1}^{L+1} \sum_{k=1}^{K_{l}} d_{m}^{2}(k)\right)
\end{gathered}
$$

WWPRD and WEDD values, of all signals from MIT-BIH Arrythmia database, were found to be less than 0.03 as indicated in Fig. 9.

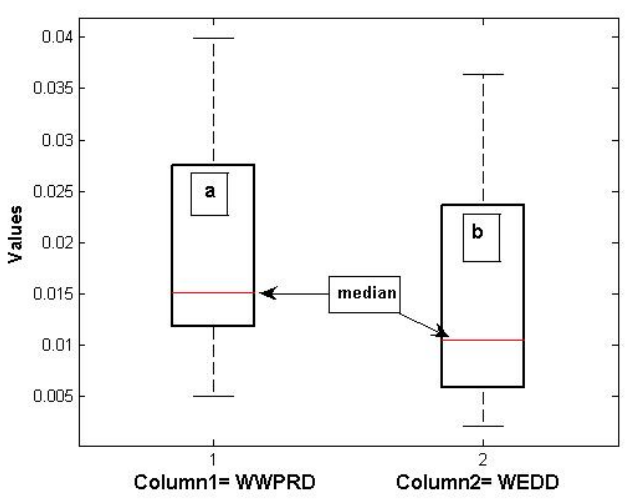

Fig.9 Boxplots of (a) WWPRD values (b) WEDD values

Cardiac arrhythmia is a heart disease where heart beats indicate change in rhythm. Three types of arrythmia were considered in our study: Normal, LBBB (Left Bundle Branch Block) and RBBB (Right Bundle Branch Block). Usually for classification purpose, features are extracted from beat to beat. In proposed algorithm features were extracted from compressed segments acquired from Modified lead II. The three records were selected from MIT-BIH Arrythmia database: Normal (103.dat), LBBB (214.dat) and RBBB (124.dat) [14]. The feature vectors were obtained from segments of above records.

Using feature vectors of 300 training segments, three cluster centers were computed by applying fuzzy c means algorithm. The ranges of feature values of compressed signal for each case are displayed in table 2 .

Table2. Ranges of feature values for each case

\begin{tabular}{|c|c|c|c|c|}
\hline \multirow{2}{*}{ Case } & $\begin{array}{c}F 1 \\
\text { minimum }\end{array}$ & $\begin{array}{c}F 2 \\
\text { maximum, }\end{array}$ & $\begin{array}{c}F 3 \\
\text { std dev. }\end{array}$ & $\begin{array}{c}F 4 \\
\text { mean }\end{array}$ \\
\hline \multirow{2}{*}{ NORMAL } & {$[-31.7$,} & {$[38.38$,} & {$[5.78$,} & {$[-0.31$,} \\
& $-15.7]$ & $92.36]$ & $13.78]$ & $0.38]$ \\
\hline \multirow{2}{*}{ LBBB } & {$[-34.77$,} & {$[20.10$,} & {$[4.56$,} & {$[0.01$,} \\
& $-29.77]$ & $26.90]$ & $4.68]$ & $0.03]$ \\
\hline \multirow{2}{*}{ RBBB } & {$[-29.90$,} & {$[15.2$,} & {$[2.66$,} & {$[-0.17$,} \\
& $-8.9]$ & $33.07]$ & $4.8]$ & $0.064]$ \\
\hline
\end{tabular}


Decision of unknown test segment was obtained by calculating Euclidean distance between its feature vector from cluster center. Minimum distance from a center declares particular class. The concerned co-ordinates of cluster center were updated.

The rows of membership functions (U) defined by equation (15) are illustrated in fig 10. Fuzzy c-Means clustering provided total classification accuracy of $97 \%$.
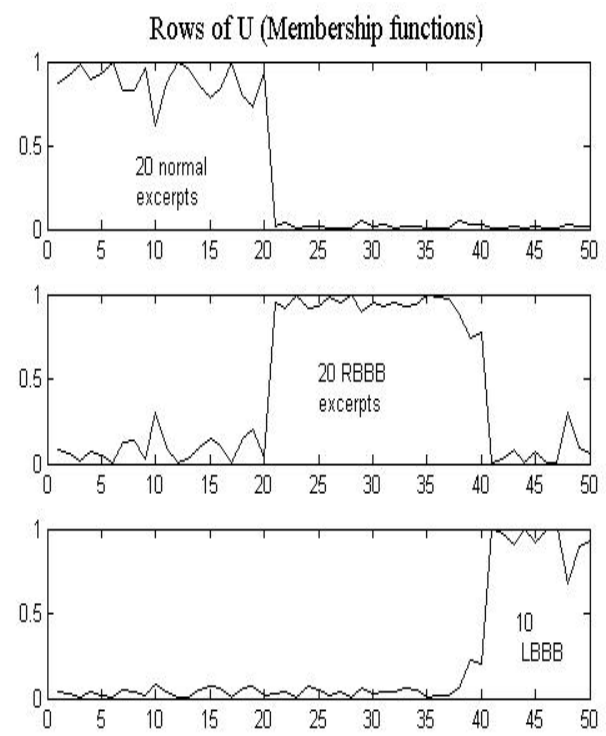

Fig.10 Membership functions of Fuzzy c Means Clustering with 20 excerpts of Normal (103.dat), 20 excerpts of RBBB (124.dat) and excerpts of LBBB (214.dat).

\section{Conclusion}

The proposed compression technique has simple steps of signal processing, avoiding complex sorting and iterative manipulation. The method can be used to compress all types of ECG signals. Evaluation of diagnostic distortion, on records of MIT-BIH Arrythmia database, proved that all clinical information was preserved after compression. Comparable classification accuracy was achieved using only four features per ECG segment, with fuzzy c means clustering. For better efficiency more training strategy should be enhanced or output of fuzzy c mean clustering may be applied to neural network. The compression algorithm requires simple hardware, three FIR filters, one subtractor and two downsampler (divide by 2), for VLSI implementation. The VLSI circuit of the proposed ECG compression will be designed in the future work.

\section{Acknowledgments}

The work was supported by funds from All India Council for Technical Education (AICTE), New Delhi under Research Promotion Scheme Grant No 8023/RID/RPS-86/2011-12.

\section{References}

[1] Yaniv Zigel, Arnon Cohen, and Amos Katz, ECG Signal Compression Using Analysis by Synthesis Coding [J]. IEEE Transactions on Biomedical Engineering, 2000, 47: 1308-1316. [2] Gulay Tohumoglu, K. Erbil Sezgin, ECG signal compression by multi-iteration EZW coding for different wavelets and thresholds [J]. Computers in Biology and Medicine, Elsevier, 2007, 37: 173-182.

[3] R. Shantha Selva Kumari, V. Sadasivam, A novelalgorithm for wavelet based ECG signal coding [J]. Computers and Electrical Engineering, Elsevier, 2007, 33:186-194.

[4] Manuel Blanco-Velasco, Fernando Cruz-Roldán ,Juan Ignacio Godino-Llorente, and Kenneth E. Barner, Wavelet Packets Feasibility Study for the Design of an ECG Compressor[J]. IEEE Transactions On Biomedical Engineering, 2007,4 : 767-781.

[5] Justin Leo Cheang Loong, Khazaimatol S Subari, Rosli Besar and Muhammad Kamil Abdullah,A New Approach to ECG Biometric Systems: A Comparitive Study between LPC and WPD Systems [A]. World Academy of Science, Engineering and Technology, 2010, 68:759-764.

[6] K.I.Ramchandran, K.P.Soman, Insight into wavelets from theory to practice [M]. Prentice Hall Ltd, Second Edition, 2006. [7] Ramchandra Manthalkar, Shubhada Ardhapurkar, Suhas Gajre,Wavelet Based ECG Denoising by Employing Cauchy Distribution at Subbands[C]. ICSP10 proceedings, 2010:17181721.

[8] Earl Gose, Richard Johnsonbaugh, Steve Jost, Pattern Recognition and Image Analysis[M]. Prentice Hall of India, 2005.

[9] Thomas Ledl, "Kernel Density Estimation: Theory and Application in Discriminant Analysis [J]. Austrian journal of statistics, 2004,43: 267-279.

[10]B.W. Silverman,Density Estimation For Statistics And Data Analysis[J]. Monographs on Statistics and Applied Probability, London: Chapman and Hall, 1986: 1-22.

[11]Website:http://www.physionet.org/physiobank/database/mi $\underline{\mathrm{tdb} /}$

[12]Gari Clifford Home page, Online Available: http://www.robots.ox.ac.uk/ gari/code.html

[13]Sabarimalai Manikandan, S. Dandapat, Wavelet energy based diagnostic distortion measure for ECG [J].Biomedical Signal Processing and Control, 2007:2 80-96.

[14] Rahime Ceylan, Yuksel Ozbay, Comparison of FCM, PCA and WT techniques for classification ECG arrhythmias using artificial neural network[J]. Expert System with Applications, ScienceDirect, 2007, 33: 286-295.

Shubhada Ardhapurkar She received Master of Engineering degree in 1994 She is currently pursuing Ph.D. in Biomedical Signal Processing Techniques from S.G.G.S. Institute of Engineering and Technology, Nanded, Maharashtra, India. Her interests include signal processing, VLSI, Communication and neural networks.

Ramchandra Manthalkar He received Ph.D. degree from Indian Institute of Technology, Kharagpur in Image Processing in 2003 and is currently working as Professor and Head in Electronics and Telecommunication Engineering Department at S.G.G.S. Institute of Engineering and Technology, Nanded. $\mathrm{He}$ has published more than 15 research papers in peer 
reviewed Journals and Conferences. He is currently guiding 6 research scholars for their Ph.D. work. Dr.Ramchandra Manthalkar's biography is included in the edition of Marquis who's who in Science and Engineering and in the International Biographical Centre of Cambridge, England as "Outstanding Scientist of $21^{\text {st }}$ century". His research interests include biomedical signal processing and image processing, analog and mixed signal VLSI Design and biometrics.

Suhas Gajre He received Ph.D. degree from Indian Institute of Technology, Delhi in Biomedical Engineering in 2007and is currently working as Associate Professor in Electronics and Telecommunication Engineering Department at S.G.G.S. Institute of Engineering and Technology, Nanded, Maharashtra, India. He has published more than 10 research papers in peer reviewed Journals and Conferences. He is currently guiding 4 research scholars for their Ph.D. work. His research interests include Biomedical Signal and Image processing, and Analog and Mixed signal VLSI Design. 